\title{
Endocarditis infecciosa asociada al cuidado de la salud. Estudio de prevalencia en un hospital de alta complejidad
}

\author{
Infective endocarditis associated with health care. Prevalence study in a high complexity \\ hospital
}

\author{
Álvaro I. Arango ${ }^{*}$, Jaime Camacho-Mackenzie², José D. Cruz-Cuevas ${ }^{3}$, Andrés F. Flórez-Monroy ${ }^{3}$, \\ Zenaida Montañez y Priscila Quintero 4
}

${ }^{1}$ Departamento de Infectología, Fundación Cardioinfantil-Instituto de Cardiología; ${ }^{2}$ Departamento de Cirugía Cardiovascular Adultos, Fundación Cardioinfantil-Instituto de Cardiología; ${ }^{3}$ Escuela de Medicina y Ciencias de la Salud, Universidad del Rosario; ${ }^{4}$ Departamento de Epidemiología, Fundación Cardioinfantil-Instituto de Cardiología. Bogotá, Colombia

\section{Resumen}

Introducción: Los procedimientos vasculares invasivos ocasionan infecciones sanguíneas asociadas a cuidados de la salud, entre ellas endocarditis infecciosa. Este evento adverso conlleva mayor morbimortalidad que la endocarditis infecciosa adquirida en la comunidad. Objetivo: Evaluar la prevalencia de endocarditis infecciosa asociada al cuidado de la salud y describir las características demográficas de esta población, así como los agentes etiológicos. Método: Estudio de corte transversal, en el que se incluyó población mayor de 18 años con endocarditis infecciosa asociada al cuidado de la salud, documentada entre enero de 2013 y junio de 2018 en la Fundación Cardioinfantil. Se realizó un análisis estadístico con distribución de variables de edad, sexo, procedimientos invasivos asociados, mortalidad y microorganismo aislado. Resultados: Se incluyeron 41 pacientes con una edad promedio de 60,6 años. No hubo distinción entre hombres (22, 53.6\%) y mujeres (19, 46.3\%). Los pacientes se agruparon, según el procedimiento realizado, en implantación de dispositivos intravasculares de alto flujo (16,39.02\%), catéter venoso central $(12,29.26 \%)$ y dispositivos intracardiacos $(11,26.82 \%)$. La prevalencia general en los procedimientos evaluados fue del $0.21 \%$, siendo del $1.42 \%$ en dispositivos intravasculares de alto flujo, del $0.72 \%$ en dispositivos intracardiacos $y$ del $0.08 \%$ en catéteres centrales. La mortalidad registrada fue del $4.9 \%$ (2 pacientes). En el $78.05 \%$ de los pacientes se obtuvo aislamiento microbiológico. Conclusiones: Los pacientes sometidos a procedimientos invasivos pueden tener un riesgo elevado de endocarditis infecciosa, conferido por el procedimiento. La baja tasa de mortalidad intrahospitalaria puede estar relacionada con la notable tasa de aislamiento positivo en hemocultivos, lo cual facilitó la terapia antibiótica dirigida.

Palabras clave: Endocarditis infecciosa. Infección nosocomial. Profilaxis antibiótica.

\section{Abstract}

Introduction: Invasive procedures the vascular tract cause health, are related to blood stream infections, among them, infective endocarditis. This adverse event leads to greater morbidity and mortality compared with community acquired infective

\section{Correspondencia:}

*Álvaro I. Arango

E-mail: alvaroignacio.arangoduque@gmail.com
Fecha de recepción: 03-04-2019

Fecha de aceptación: 09-12-2019

DOI: 10.24875/RCCAR.M21000049
Disponible en internet: 29-07-2021 Rev Colomb Cardiol. 2021;28(3):239-245 www.rccardiologia.com 0120-5633 / @ 2020 Sociedad Colombiana de Cardiología y Cirugía Cardiovascular. Publicado por Permanyer. Este es un artículo open access bajo la licencia CC BY-NC-ND (http://creativecommons.org/licenses/by-nc-nd/4.0/). 
endocarditis. Objective: To evaluate the prevalence of healthcare-associated infective endocarditis, describe the demographic characteristics of this population and the etiological agents. Method: Cross-sectional descriptive, prevalence study of prevalence. It included patients $\geq 18$ years old with healthcare-associated infective endocarditis, documented at Fundación Cardioinfantil. Statistical analysis with distribution of variables of age, gender, invasive procedure associated and isolated microorganism was made. Results: 41 patients were obtained. The average age was 60.6 years. There was no distinction between men (22, 53.6\%) and women (19, 46.3\%). The patients were grouped according to the procedure performed in: implantation of high-flow intravascular devices (16, 39.02\%), central venous catheter (12,29.26\%) and intracardiac devices $(11,26.82 \%)$. The general prevalence in the evaluated procedures was $0.21 \%$, being $1.42 \%$ in high-flow intravascular devices, $0.72 \%$ in intracardiac devices and $0.08 \%$ in central catheters. The registered mortality was $4,9 \%$ (2 patients). In $78.05 \%$ of the patients, microbiological isolation was obtained. Conclusions: patients who has invasive procedures may have an incremented risk of infective endocarditis because of the procedure. The low intrahospital mortality could be related with the remarkable number of microbiological identification which facilitated a directed antimicrobial therapy.

Key words: Infective endocarditis. Nosocomial infection. Antibiotic prophylaxis.

\section{Introducción}

Partiendo del principio de que la bacteriemia es generada por la translocación y la invasión del torrente sanguíneo por la microbiota humana (oral, intestinal y piel), se sabe que durante los procedimientos invasivos o la colocación de dispositivos vasculares, en los que se genera una disrupción de las barreras epiteliales 0 mucosas, es posible ocasionar una bacteriemia transitoria ${ }^{1,2}$. Este fenómeno puede llevar a infecciones sanguíneas asociadas al cuidado de la salud, las cuales son reconocidas como procesos nosocomiales con una alta morbimortalidad ${ }^{3}$. Las infecciones originadas en el ámbito hospitalario muestran un crecimiento exponencial en los pacientes con riesgo de esta categoría, particularmente en centros de alta complejidad con una elevada tasa de procedimientos y dispositivos invasivos $^{4}$. En ocasiones, dicha bacteriemia logra una siembra y replicación sobre la superficie endocárdica para formar una vegetación adherente a una válvula nativa o protésica, condición conocida como endocarditis infecciosa, la cual es infrecuente, pero potencialmente mortal, además de tener una alta incidencia de complicaciones entre los sobrevivientes ${ }^{5}$.

La endocarditis infecciosa asociada al cuidado de la salud es una complicación grave de la atención en salud, que muestra una creciente incidencia en la literatura y representa alrededor del $30 \%$ de todos los casos de endocarditis infecciosa ${ }^{6-8}$, e incluso en algunas series se ha reportado más frecuente que la endocarditis infecciosa adquirida en la comunidad ${ }^{7,9}$. Este aumento en la incidencia se ha relacionado en algunos trabajos con una disminución en la realización de profilaxis antibiótica ${ }^{10-14}$. Empeorando el panorama, en otros estudios la endocarditis infecciosa asociada al cuidado de la salud ha mostrado tener mayores morbilidad y mortalidad en comparación con la adquirida en la comunidad ${ }^{15}$.

Varios estudios realizados en diferentes países durante las últimas décadas han sugerido que las características de la endocarditis han cambiado tanto en su componente microbiológico como en el perfil demográfico. Dichos cambios se han evidenciado principalmente en la endocarditis infecciosa asociada al cuidado de la salud, lo cual podría estar relacionado con procedimientos médico-quirúrgicos invasivos ${ }^{7,9,14,16-20}$.

En Colombia, los datos sobre la endocarditis infecciosa asociada al cuidado de la salud son muy escasos y con una cantidad de pacientes muy pequeña. Solo existe un estudio en el que se documenta esta condición en 144 pacientes, de los cuales el 6\% se habían sometido a procedimientos invasivos en la Fundación Cardioinfantil-Instituto de Cardiología ${ }^{21}$. Otro estudio realizado en Colombia reporta 105 pacientes con endocarditis infecciosa sin mención de asociación a intervenciones médico-quirúrgicas ${ }^{22}$. Por último, Madrid et al. ${ }^{23}$ reportan 104 casos sin mencionar directamente la implicación de intervenciones de cuidados de la salud. En este último estudio $^{23}$ se describe que la enfermedad valvular degenerativa, la implantación de dispositivos endovasculares y las prótesis valvulares han reemplazado a la fiebre reumática como principal situación de riesgo para adquirir endocarditis infecciosa.

De acuerdo con lo anterior, es evidente la necesidad de caracterizar la epidemiología actual de la endocarditis infecciosa asociada al cuidado de la salud, su relación con procedimientos invasivos médico-quirúrgicos específicos y las características microbiológicas y demográficas de esta afección, buscando dar un paso hacia el futuro para la identificación de factores predictores específicos que permitan anticipar las complicaciones y determinar esquemas terapéuticos y profilácticos. Con 
esto, el objetivo del presente estudio fue evaluar la prevalencia de endocarditis infecciosa asociada al cuidado de la salud en pacientes mayores de 18 años sometidos a procedimientos invasivos en la Fundación Cardioinfantil-Instituto de Cardiología entre enero de 2013 y junio de 2018, y describir las características demográficas de esta población, el tipo de válvula afectada, la mortalidad y los agentes etiológicos.

\section{Método}

Se presenta un estudio observacional de corte transversal. Se utilizó la base de datos del comité de infecciones de la Fundación Cardioinfantil para obtener los datos de interés. En el estudio se incluyeron pacientes mayores de 18 años, atendidos entre enero de 2013 y junio de 2018, con diagnóstico intrahospitalario de endocarditis infecciosa conforme a los criterios de Duke modificados ${ }^{11}$. Posteriormente, de esta población se seleccionaron únicamente aquellos pacientes que, previo al diagnóstico de endocarditis asociada al cuidado de la salud, fueron sometidos a un procedimiento invasivo realizado en la institución durante la misma hospitalización en la que se presentó la complicación infecciosa mencionada.

Una vez recopilados los datos, se tabuló la información demográfica, clínica y microbiológica de la población. Los datos registrados fueron sometidos a análisis estadístico con el software de Microsoft Excel para obtener la distribución de las siguientes variables: edad en años al momento del diagnóstico, sexo (hombre o mujer), procedimiento invasivo asociado, estructura cardiaca afectada según los hallazgos imagenológicos (válvula nativa, válvula protésica o estructura no valvular), resultado de los hemocultivos (negativo, positivo y microorganismo aislado) y mortalidad intrahospitalaria.

Además, se revisaron las bases de datos de la institución para determinar el número de veces que se realizaron los procedimientos relacionados durante el periodo analizado, y de esta forma poder calcular la prevalencia de la infección según cada procedimiento. Los procedimientos relacionados se agruparon en tres categorías principales: implantación de dispositivos intravasculares de alto flujo (cambio valvular aórtico, cambio valvular mitral y reconstrucción de aorta ascendente), implantación de dispositivos intracardiacos (marcapasos y cardiodesfibrilador implantable) y colocación de catéteres centrales. Una vez calculada la prevalencia de cada categoría se realizó el cálculo de la razón de prevalencia de los dos primeros con la prevalencia de los pacientes con catéter, con un intervalo de confianza del 95\% (IC95\%), y se calculó el valor de $p$ por medio de la prueba de Mantel-Haenszel. Se utilizó el software Epi-Info de libre acceso.

\section{Resultados}

En la búsqueda en la base de datos de la institución se obtuvo una población de 41 pacientes con endocarditis infecciosa asociada al cuidado de la salud que cumplían los criterios de inclusión en el periodo evaluado. El promedio de edad al momento del diagnóstico fue de 60.6 años (desviación estándar: 19.6 años). No hubo una diferencia significativa entre sexos: 22 hombres $(53.6 \%)$ y 19 mujeres (46.3\%) (Tabla 1$)$.

La distribución de los procedimientos entre la población fue heterogénea, con un 39.02\% (16 de 41) de los casos relacionados con la implantación de dispositivos intravasculares de alto flujo, seguidos de catéter venoso central en el $29.26 \%$ (12 de 41) e implantación de dispositivos intracardiacos en el $26.82 \%$ (11 de 41). Los dos casos restantes correspondieron a una vertebroplastia y una ligadura de várices esofágicas, representando el $2.43 \%$ cada uno (Tabla 1); estos dos casos no fueron considerados para el cálculo de la prevalencia debido a la ausencia de datos necesarios para un análisis completo, por lo que la prevalencia se calculó con los 39 procedimientos restantes. De acuerdo con la clasificación de las intervenciones antes mencionadas, la prevalencia general de endocarditis infecciosa asociada al cuidado de la salud fue del $0.21 \%$, mientras que determinada por cada grupo en particular se calculó lo siguiente: $1.42 \%$ para dispositivos intravasculares de alto flujo, $0.72 \%$ para dispositivos intracardiacos y $0.08 \%$ para catéteres centrales (Tabla 2). Al comparar la prevalencia de los dos primeros subgrupos con el subgrupo de catéter (menor prevalencia encontrada), se encontró una razón de prevalencia de 9.38 (IC95\%: 4.14-21.23; $p<0.001)$ y $18.49(8.77-38.99 ; p<0.001)$ para dispositivos intracardiacos y para dispositivos intravasculares de alto flujo, respectivamente.

El tipo de estructura cardiaca afectada fue una válvula nativa en 18 casos (43.9\%), una válvula protésica en 13 casos (31.7\%) y una estructura no valvular en 10 casos $(24.4 \%)$, sin relacionarse esta diferencia con ningún procedimiento específico. A todos los pacientes se les realizaron hemocultivos, de los cuales el $34.14 \%$ correspondieron a Staphylococcus aureus (14 casos), el $12.19 \%$ a enterobacterias (2 Escherichia coli, 1 Salmonella spp., 1 Enterococcus cloacae, 1 Proteus mirabilis), el $12.19 \%$ a estafilococos coagulasa negativos 
Tabla 1. Características demográficas

\begin{tabular}{|l|c|c|c|c|c|}
\hline \multirow{2}{*}{ Grupo } & \multirow{N}{*}{$N(\%)$} & \multicolumn{2}{|c|}{ Edad al diagnóstico (años) } & \multicolumn{2}{|c|}{ Sexo } \\
\cline { 3 - 6 } & & Mediana & Rango intercuatílico & Hombres & Mujeres \\
\hline DIC & $11(26.82 \%)$ & 66 & $51.5-76.5$ & $6(54.54 \%)$ & $5(45.45 \%)$ \\
\hline DIVAF & $16(39.02 \%)$ & 61 & $38-72.25$ & $10(62.5 \%)$ & $6(37.5 \%)$ \\
\hline Catéter & $12(29.26 \%)$ & 66 & $53.5-75.5$ & $5(41.6 \%)$ & $7(58.3 \%)$ \\
\hline Todos* & $41(100 \%)$ & 63 & $52-74$ & $22(53.6 \%)$ & $19(46.3 \%)$ \\
\hline
\end{tabular}

DIC: dispositivos intracardiacos (marcapasos y cardiodesfibrilador implantable); DIVAF: dispositivos intravasculares de alto flujo (cambio valvular aórtico, cambio valvular mitral, reconstrucción de aorta). *Incluyen un caso de ligadura de várices esofágicas y una vertebroplastia.

Tabla 2. Prevalencia total y por subgrupos de endocarditis infecciosa asociada al cuidado de la salud posterior a procedimientos invasivos en el periodo evaluado

\begin{tabular}{|l|c|c|c|c|}
\hline Procedimiento & Cantidad & Endocarditis & Prevalencia & Razón de prevalencia* (IC95\%) $\mathbf{p}^{\dagger}$ \\
\hline DIC & 1.528 & 11 & $0.72 \%$ & $9.38(4.14-21.23) \mathrm{p}<0.001$ \\
\hline CDI & 568 & 7 & $1.23 \%$ & \\
\hline Marcapasos & 960 & 4 & $0.42 \%$ & $18.49(8.77-38.99) \mathrm{p}<0.001$ \\
\hline DIVAF & 1.128 & 16 & $1.42 \%$ & $1.02 \%$ \\
\hline Cambio valvular aórtico & 783 & 8 & $1.90 \%$ & \\
\hline Cambio valvular mitral & 316 & 6 & $6.90 \%$ & \\
\hline Reconstrucción de aorta & 29 & 2 & $0.08 \%$ & \\
\hline Catéteres & 15.645 & 12 & $1.02 \%$ & \\
\hline Prevalencia sin catéteres & 2.656 & 27 & $0.21 \%$ & \\
\hline Total & 18.301 & 39 & &
\end{tabular}

CDI: cardiodesfibrilador implantable; DIC: dispositivos intracardiacos; DIVAF: dispositivos intravasculares de alto flujo; IC95\%: intervalo de confianza del 95\%. *Cálculo de la razón de prevalencia entre la prevalencia de cada categoría y la calculada para pacientes con colocación de catéter.

${ }^{\dagger}$ Prueba de Mantel-Haenszel.

(4 Staphylococcus epidermidis, 1 Staphylococcus hominis), el $9.75 \%$ a Candida spp. (2 Candida glabrata, 1 Candida lusitaniae, 1 Candida albicans), el 7.31\% a Pseudomonas aeruginosa ( 3 casos) y el $2.41 \%$ a Enterococcus faecalis (1 caso). Por otro lado, el $21.95 \%$ de los casos no tuvieron aislamiento microbiológico (9 casos) (Fig. 1).

\section{Discusión}

Teniendo en cuenta la población de pacientes de la Fundación Cardioinfantil con endocarditis infecciosa asociada al cuidado de la salud posterior a la realización de procedimientos invasivos en los que no se realiza profilaxis antibiótica para prevenir esta complicación, se presentó una prevalencia general del $0.21 \%$ en el periodo evaluado, lo cual es 14 veces mayor que la prevalencia en la población general, que se estima en un $0.015 \%{ }^{12}$. La prevalencia reportada en este estudio es similar a la relacionada con el reemplazo valvular aórtico transcatéter reportada por Yeo et al. ${ }^{24}$, la cual fue del $0.3 \%$, con una mortalidad intrahospitalaria del $20.8 \%$. Este grupo en particular, facilitado por factores de riesgo específicos, podría estar contribuyendo a la tendencia actual de una incidencia creciente, en particular posterior a los ajustes en la profilaxis antibiótica ${ }^{13,14,19}$.

Al evaluar por subgrupos según el procedimiento asociado, se encontró una prevalencia mayor para los dispositivos intracardiacos (cardiodesfibrilador implantable o marcapasos) y los dispositivos intravasculares de alto flujo, en comparación con los pacientes a 


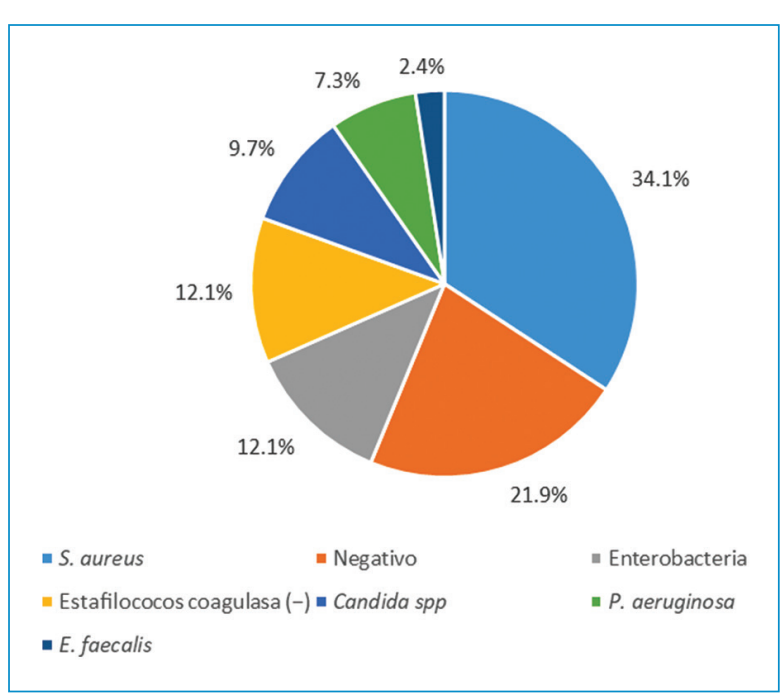

Figura 1. Resultados de los hemocultivos. La mortalidad de la población aquí expuesta fue muy baja; de los 41 pacientes, tan solo dos murieron durante la estancia hospitalaria, lo que representa una mortalidad del $4.9 \%$.

quienes se les colocaron catéteres. Este fenómeno ya se documentó, en particular en los casos de endocarditis infecciosa asociada a dispositivos implantables, en el trabajo de Athan et al. ${ }^{25}$, en el cual identificaron 177 pacientes con esta complicación, correspondientes al $6.4 \%$ de la cohorte. El $45.8 \%$ de los casos fueron infecciones asociadas al cuidado de la salud y el microorganismo predominante fue $S$. aureus. Lo anterior es perfectamente predecible por la habilidad de $S$. aureus de adherirse a células eucariotas y a materiales abióticos mediante sus proteínas de la pared celular, con la subsecuente producción de biopelícula como importante factor de virulencia, en la particular situación de patogenia que involucra la endocarditis infecciosa asociada al cuidado de la salud.

Por otro lado, Janszky et al..$^{10}$ realizaron un estudio que incluyó más de 7000 pacientes, usando un modelo de casos y controles, y lograron identificar una asociación entre el desarrollo de endocarditis infecciosa y haberse sometido a algún procedimiento invasivo en las 12 semanas previas, incluyendo procedimientos cardiovasculares (en particular bypass coronario), procedimientos en la piel y manejo de heridas, transfusión, diálisis, aspirado de médula ósea y procedimientos endoscópicos.

La mediana de edad de la población estudiada se encuentra alrededor de los 60 años, lo cual concuerda con la tendencia al aumento en la edad de presentación de esta enfermedad según lo reportado en series anteriores $9,16,20,26$. La distribución por sexo de los pacientes con endocarditis infecciosa asociada al cuidado de la salud tiende a ser más homogénea (hombre: mujer 1.2: 1), a diferencia de la endocarditis infecciosa adquirida en la comunidad (1.7:1 $)^{27,28}$. Esto se puede deber a que los factores predisponentes, derivados de los procedimientos intervencionistas y del implante de material protésico, son independientes del sexo. Al evaluar las variables de edad y sexo en los subgrupos establecidos, no se encontraron diferencias significativas.

En cuanto a los microorganismos causales, S. aureus representó en la serie el $34 \%$ de los aislados. Esta es una distribución similar a la de otros estudios (20$36.5 \%)^{15,29}$. Sin embargo, Giannitsioti et al. ${ }^{30}$, Francischeto et al. ${ }^{31}$, Martín-Dávila et al. ${ }^{32}$ y Reji et al. ${ }^{28}$ tuvieron tasas más bajas de $S$. aureus, entre el $11 \%$ y el $20 \%$. Los estafilococos coagulasa negativos representaron el $12 \%$ de los aislamientos, lo cual, comparado con otros estudios, se ubica en el límite inferior de lo informado, que oscila entre el $11 \%$ y el $27.6 \% 15,29,31$. El grupo de pacientes presentado en este estudio difiere considerablemente en la distribución etiológica por enterobacterias, que ascendió al $12 \%$, en contraparte de lo informado por Francischetto et al. ${ }^{31}(4 \%)$ y Lomas et al. ${ }^{15}(4.7 \%)$, pero concuerda con lo reportado por Reji et al. ${ }^{28}$. Otra gran diferencia se observa en E. faecalis, con un porcentaje del $3 \%$ de los casos, muy por debajo de lo reportado en Brasil, España e India, con un $19 \%$, un $17.3 \%$ y un $6 \%$, respectivamente ${ }^{31,15,28}$. La distribución porcentual de las levaduras fue del $10 \%$, muy similar a la serie brasilera ${ }^{31}(13 \%)$, mientras que en España ${ }^{15}$ solo representó el 3.1\%. Las diferencias que se perciben entre los microorganismos implicados en el presente estudio y los reportados previamente podrían estar asociadas, en parte, a la variación microbiológica de acuerdo con el área geográfica, lo cual reitera la importancia de documentar y conocer la flora específica global, regional, local e institucional.

En lo referente a la estructura cardiaca afectada, se observó una ligera predominancia de la válvula nativa respecto a la válvula protésica; sin embargo, en las series previas esta diferencia suele ser más marca$\mathrm{da}^{9,28}$. En concreto, al tratarse de pacientes con endocarditis asociada al cuidado de la salud y dispositivos cardiacos implantables, se genera un tercer grupo significativo que corresponde a vegetaciones en estructuras no valvulares, como la cablitis, que en este caso correspondió al $24.4 \%$, entendido bajo el contexto de 
los factores de virulencia de algunos cocos grampositivos, como la biopelícula ${ }^{33}$.

Se documentó una mortalidad intrahospitalaria de tan solo el $4.9 \%$, que comparada con las cifras de otras latitudes, como Brasil, es muy baja, pues en este país se presentó una mortalidad de hasta el $32 \%{ }^{31}$. Así mismo, en España ascendió al $44.9 \%$ en un total de 127 pacientes, llegando incluso al $83.3 \%$ cuando la endocarditis infecciosa asociada al cuidado de la salud no se intervino quirúrgicamente ${ }^{15}$. Además, en un análisis multivariado, el estudio de España confirmó una mortalidad mayor en los casos de endocarditis infecciosa asociada al cuidado de la salud exclusivamente en una válvula nativa, con un índice de riesgo de incidencia de 1.20 (IC95\%: 1.03-1.61) ${ }^{29}$. Del mismo modo, en la serie de Lin et al. ${ }^{9}$, en el subgrupo de endocarditis infecciosa asociada al cuidado de la salud la mortalidad ascendió al 73.1\%. La baja mortalidad en el presente estudio puede relacionarse con la alta tasa de aislamiento del agente etiológico, alrededor del $80 \%$, lo que permitió instaurar una terapia antimicrobiana dirigida en lugar de empírica, lo cual mejora los desenlaces clínicos robustos, como la mortalidad. En el estudio realizado por Cahill et al. ${ }^{6}$ con pacientes con endocarditis infecciosa y cardiopatías congénitas hubo, de modo similar a este estudio, una alta tasa de aislamiento microbiológico (88\%), que también se relacionó con una baja mortalidad intrahospitalaria (6.7\%). Así, se ha visto que, a pesar de una antibioticoterapia empírica dirigida hacia los patógenos más frecuentes según el subtipo de pacientes, en alrededor del $50 \%$ de los casos en los que se obtiene aislamiento microbiológico se requiere un ajuste del tratamiento antibiótico, bien sea para disminuir o ampliar el espectro ${ }^{28}$. Esto podría sugerir que es necesario actualizar el conocimiento referente al perfil microbiano asociado a esta condición y replantear el espectro de la terapia empírica, en caso de ser necesario. Otros factores identificados previamente, que se asocian con mayor mortalidad en los pacientes con endocarditis infecciosa asociada al cuidado de la salud, comparada con la adquirida en la comunidad, son la mayor edad, el uso de catéteres vasculares, la hemodiálisis, la infección por $S$. aureus resistente a la meticilina, la cirrosis, el cáncer y la quimioterapia ${ }^{34}$.

Entre las limitaciones del estudio se encuentra el hecho de ser observacional y de corte transversal, lo cual limitó el seguimiento posterior al egreso de los pacientes y el conocimiento de otros desenlaces de interés, como complicaciones y manejo quirúrgico. Así mismo, existe un sesgo al tratarse de un estudio unicéntrico, con un tamaño de muestra limitado que dificulta la comparación estadística del número de eventos por subgrupo y la exclusión de dos casos en el análisis de la prevalencia por no tener datos suficientes. Debido a las características del estudio no se puede afirmar una relación de causalidad entre los procedimientos mencionados y el desarrollo de endocarditis infecciosa, pero los resultados sugieren una asociación entre estas dos variables. Para resolver estas limitaciones se requieren estudios con mayor robustez estadística, centrados en la prevalencia y la incidencia de endocarditis infecciosa asociada al cuidado de la salud, su relación con los distintos procedimientos e intervenciones invasivas, y el papel de la profilaxis antibiótica. Del mismo modo, es relevante aumentar los estudios que caractericen los microorganismos implicados en la epidemiología nacional, regional, local e institucional.

\section{Conclusiones}

En los pacientes que fueron sometidos a procedimientos invasivos, en particular la colocación de dispositivos intracardiacos y de dispositivos intravasculares de alto flujo, se documentó una elevada prevalencia de endocarditis infecciosa al compararla con la reportada en la población general. Lo anterior indica que estos pacientes pueden tener un riesgo elevado de endocarditis infecciosa conferido por el procedimiento. Desde el punto de vista fisiopatológico, este hecho se podría explicar por la bacteriemia generada al lesionar las barreras mecánicas, sumado a la alteración en la estructura cardiaca con material abiótico que facilita la adhesión bacteriana. El aumento en la prevalencia de esta infección con mal pronóstico puede estar relacionado con un incremento en el número de procedimientos y de dispositivos invasivos, los cuales se comportan como factores de riesgo. Así mismo, el aumento de la edad promedio de presentación y la distribución igual entre sexos son congruentes con el progresivo cambio en las características de la población afectada. Finalmente, la baja tasa de mortalidad intrahospitalaria puede estar relacionada con la notable tasa de aislamiento positivo en hemocultivos, que facilitó la terapia antibiótica dirigida, recalcando la importancia de tomar muestras idóneas para determinar el microorganismo causal y hacer un ajuste en la terapia.

\section{Conflicto de intereses}

No existe conflicto de intereses por parte de ninguno de los autores en el presente artículo. 


\section{Responsabilidades éticas}

Protección de personas y animales. Los autores declaran que para esta investigación no se han realizado experimentos en seres humanos ni en animales.

Confidencialidad de los datos. Los autores declaran que han seguido los protocolos de su centro de trabajo sobre la publicación de datos de pacientes.

Derecho a la privacidad y consentimiento informado. Los autores declaran que en este artículo no aparecen datos de pacientes.

\section{Bibliografía}

1. Christaki E, Giamarellos-Bourboulis EJ. The complex pathogenesis of bacteremia. Virulence. 2014;5:57-65. [Citado 30 julio 2019]. Disponible en: http://www.tandfonline.com/doi/abs/10.4161/viru.26514.

2. Smith DA, Nehring SM. Bacteremia. StatPearls. 2019. [Citado 30 julio 2019]. Disponible en: http://www.ncbi.nlm.nih.gov/pubmed/28723008.

3. Brady M, Oza A, Cunney R, Burns K. Attributable mortality of hospital-acquired bloodstream infections in Ireland. J Hosp Infect. 2017;96:35-41. Disponible en: https://linkinghub.elsevier.com/retrieve/pii/S0195670117300920.

4. Ávila-Puentes M. Boletín Epidemiológico. Infecciones Asociadas a la Atención en Salud 2013-2014. Secretaría Distrital de Salud. 2014. Disponible en: http://theific.org/wp-content/uploads/2014 / 08/Spanish_ch3_ PRESS.pdf.

5. Park LP, Chu VH, Peterson G, Skoutelis A, LejkonZupa T, Bouza E, et al. Validated Risk Score for Predicting 6ロMonth Mortality in Infective Endocarditis. J Am Heart Assoc. 2016;5:e003016. [Citado 29 julio 2019]. Disponible en: http://www.ncbi.nlm.nih.gov/pubmed/27091179.

6. Cahill T, Jewell P, Denne L, Franklin R, Frigiola A, Orchard E, et al. Contemporary epidemiology of infective endocarditis in patients with congenital heart disease: a UK prospective study. Am Heart J. 2019;215:70-7. Disponible en: https://doi.org/10.1016/j.ahj.2019.05.014.

7. Camou F, Dijos M, Barandon L, Cornolle C, Greib C, Laine M, et al. Management of infective endocarditis and multidisciplinary approach. Médecine Mal Infect. 2019;49:17-22. Disponible en: https://doi.or$\mathrm{g} / 10.1016 / \mathrm{j} . \mathrm{medmal}$.2018.06.007.

8. Muñoz P, Kestler M, De Alarcon A, Miro JM, Bermejo J, Rodríguez-Abe$\mathrm{Ila} \mathrm{H}$, et al. Current epidemiology and outcome of infective endocarditis. Medicine (Baltimore). 2015;94:e1816. Disponible en: https://insights.ovid. com/crossref?an=00005792-201510270-00037.

9. Lin AN, Kyaw H, Lin K, Pendharkar S, Shaikh AZ, Ayala-Rodriguez C, et al. Trends in epidemiology: analysis of risk factors and outcomes of infective endocarditis. A retrospective study (2009-2015). Cureus. 2019;11:1-8. Disponible en: https://www.cureus.com/articles/17242-trends-in-epidemiology-analysis-of-risk-factors-and-outcomes-of-infective-endocarditis-a-retrospective-study-2009-2015.

10. Janszky I, Gémes K, Ahnve S, Asgeirsson H, Möller J. Invasive procedures associated with the development of infective endocarditis. J Am Coll Cardiol. 2018;71:2744-52. Disponible en: https://linkinghub.elsevier. com/retrieve/pii/S0735109718345522.

11. Habib G, Lancellotti P, Antunes MJ, Bongiorni MG, Casalta J-P, Del Zotti F, et al. 2015 ESC Guidelines for the management of infective endocarditis. Eur Heart J. 2015;36:3075-128. Disponible en: https://academic.oup.com/eurheartj/article-lookup/doi/10.1093/eurheartj/ehv319.

12. Keller K, von Bardeleben RS, Ostad MA, Hobohm L, Munzel T, Konstantinides $\mathrm{S}$, et al. Temporal trends in the prevalence of infective endocarditis in Germany between 2005 and 2014. Am J Cardiol. 2017;119:31722. [Citado 29 julio 2019]. Disponible en: http://www.ncbi.nlm.nih.gov/ pubmed/27816113.

13. Dayer $M$, Jones $S$, Prendergast $B$, Baddour LM, Lockhart PB Thornhill $\mathrm{MH}$. An increase in the incidence of infective endocarditis in England since 2008: a secular trend interrupted time series analysis. Lancet. 2017;385:1219-28.

14. Van Den Brink FS, Swaans MJ, Hoogendijk MG, Alipour A, Kelder JC Jaarsma W, et al. Increased incidence of infective endocarditis after the 2009 European Society of Cardiology guideline update: a nationwide study in the Netherlands. Eur Hear J - Qual Care Clin Outcomes. 2017:3:141-7.

15. Lomas JM, Martínez-Marcos FJ, Plata A, Ivanova R, Gálvez J, Ruiz J, et al. Healthcare-associated infective endocarditis: an undesirable effect of healthcare universalization. Clin Microbiol Infect. 2010;16:1683-90. Disponible en: https://linkinghub.elsevier.com/retrieve/pii/S1198743X14605668.
16. Joffre J, Dumas G, Aegerter P, Dubée V, Bigé N, Preda G, et al. Epidemiology of infective endocarditis in French intensive care units over the 1997-2014 period - from CUB-Réa Network. Crit Care. 2019;23:143. Disponible en: https://ccforum.biomedcentral.com/articles/10.1186/ s13054-019-2387-8.

17. Farah A, Ben Abdeljelil O, Jomaa W, Gana A, Ben Hamda K, Maatouk F. Infective endocarditis: a changing epidemiological profile over the years. A prospective cohort study. Arch Cardiovasc Dis Suppl. 2019;11:e316-7. Disponible en: http://dx.doi.org/10.1016/j.acvdsp.2019.04.026.

18. Cecchi E, Chirillo F, Castiglione A, Faggiano P, Cecconi M, Moreo A, et al. Clinical epidemiology in Italian Registry of Infective Endocarditis (RIEI): focus on age, intravascular devices and enterococci. Int J Cardiol. 2015;190:151-6. Disponible en: https://linkinghub.elsevier.com/retrieve/ pii/S0167527315008463.

19. Chirillo F, Faggiano P, Cecconi M, Moreo A, Squeri A, Gaddi O, et al Predisposing cardiac conditions, interventional procedures, and antibiotic prophylaxis among patients with infective endocarditis. Am Heart J. 2016:179:42-50. Disponible en: http://dx.doi.org/10.1016/j.ahj.2016.03.028.

20. Noureddine-López M, de la Torre-Lima J, Ruiz-Morales J, Gálvez-Acebal J, Hidalgo-Tenorio C, de Alarcón González CA. Endocarditis infecciosas izquierdas, cambios con el nuevo milenio. Med Clin (Barc). 2019;153:636. Disponible en: https://doi.org/10.1016/j.medcli.2018.04.018.

21. Noreña E, Arango A, Marin F, Berltra J. Caracterización clínica, microbiológica y ecocardiográfica de pacientes con endocarditis infecciosa en la Fundación Cardioinfantil 2002-2012. Disponible en: http://repository. urosario.edu.co/handle/10336 / 4976.

22. Echeverria R, Rozo J, Jaramillo C, Rodríguez C. Características epidemiológicas de la endocarditis infecciosa en la Fundación Clínica A. Shaio entre 1994-2001. Rev Colomb Cardiol. 2002;10:59-63.

23. Madrid CA, Jaramillo AN, Roncancio G, Mauricio J, Pérez G, Franco L, et al. Epidemiología de la endocarditis infecciosa en pacientes adultos atendidos en un centro cardiovascular de Colombia, experiencia de 10 años. Rev Esp Cardiol. 2014;67:914.

24. Yeo I, Kim LK, Park SO, Wong SC. In-hospital infective endocarditis following transcatheter aortic valve replacement: a cross-sectional study of the National Inpatient Sample database in the USA. J Hosp Infect. 2018;100:444-50. Disponible en: https://doi.org/10.1016/j.jhin.2018.05.014.

25. Athan E, Chu VH, Tattevin P, Selton-Suty C, Jones $P$, Naber $C$, et al. Clinical characteristics and outcome of infective endocarditis involving implantable cardiac devices. JAMA. 2012;307:1727. Disponible en: http:// jama.jamanetwork.com/article.aspx?doi=10.1001/jama.2012.497.

26. Flouriot C, Avinee G, Joulakian M, Alarçon C, Marchand C, Savouré A et al. Infective endocarditis after transcatheter aortic valve implantation. A comparison with endocarditis occurring in surgical aortic prosthesis and native aortic valve patients. Arch Cardiovasc Dis Suppl. 2019;11:246. Disponible en: https://linkinghub.elsevier.com/retrieve/pii/S1878648019301910.

27. Fowler VJ, Scheld W, Bayer A. Endocarditis and intravascular infections. En: Mandell GL, Bennett JE, Dolin R, editores. Principles and practice of infectious diseases. $7^{\text {th }}$ ed. Philadelphia: Elsevier Churchill Livingstone; 2009.

28. Reji R, Thomas A, Rajappan A, Joseph S, Reghu R. A Prospective study to analyse the agreement between empirical antimicrobials used in infective endocarditis and culture sensitivity reports. J Clin Diagnostic Res. 2019;13:5-9.

29. Benito N, Miró JM, De Lazzari E, Cabell CH, Del Río A, Altclas J, et al.; International Collaboration on Endocarditis Prospective Cohort Study (ICE-PCS) Investigators. Health care-associated native valve endocarditis in patients with no history of injection drug use: current importance of non-nosocomial acquisition. Ann Int Med. 2009;150:586-94. Disponible en: https://www.ncbi.nlm.nih.gov/pmc/articles/PMC3625649/pdf/nihms451194.pdf.

30. Giannitsioti E, Skiadas I, Antoniadou A, Tsiodras S, Kanavos K, Triantafyllidi H, et al. Nosocomial vs. community-acquired infective endocarditis in Greece: changing epidemiological profile and mortality risk. Clin Microbiol Infect. 2007;13:7639. Disponible en: http://dx.doi.org/10.1111/j.1469-0691.2007.01746.x

31. Francischetto $O$, da Silva LAP, Senna KMS, Vasques MR, Barbosa GF Weksler C, et al. Healthcare-associated infective endocarditis: case series in referral hospital from 2006 to 2011. Arq Bras Cardiol. 2014;103:292-8. Disponible en: http://www.gnresearch.org/doi/10.5935/ abc.20140126.

32. Martín-Dávila P, Fortún J, Navas E, Cobo J, Jiménez-Mena M, Moya JL, et al. Nosocomial endocarditis in a tertiary hospital. Chest. 2005;128:7729. Disponible en: https://linkinghub.elsevier.com/retrieve/pii/ S0012369215504248.

33. Garrett TR, Bhakoo M, Zhang Z. Bacterial adhesion and biofilms on surfaces. Prog Nat Sci. 2008;18:1049-56. Disponible en: https://linkinghub.elsevier.com/retrieve/pii/S1002007108002049.

34. Hwang J, Park SW, Cho EJ, Lee GY, Kim EK, Chang S-A, et al. Risk factors for poor prognosis in nosocomial infective endocarditis. Korean $\mathrm{J}$ Intern Med. 2018;33:102-12. Disponible en: http://kjim.org/journal/view. php?doi=10.3904/kjim.2016.106. 